\title{
Structure and transformation Clusters of molecular liquids and solids
}

\author{
Lawrence S. Bartell \\ Department of Chemistry, University of Michigan, Ann Arbor, MI 48109, USA
}

(Received 21 August 1993; accepted 15 November 1993)

\begin{abstract}
Molecular dynamics simulations of structural and dynamic characteristics of molecular clusters are proving to be a valuable aid in the interpretation of properties of molecular liquids and solids. The role of clusters as computationally tractable models of condensed matter undergoing phase changes is analyzed.
\end{abstract}

\section{Introduction}

Molecular dynamics (MD) simulations of clusters have been applied extensively to gain insight into the behavior of molecules in condensed phases and, especially, in phase changes. Almost all of the MD studies of clusters to date, however, have been on atomic clusters, principally on clusters of Lennard-Jones spheres [1]. Studies of clusters in this laboratory, on the other hand, have focused on systems of polyatomic molecules $[2,3]$. It is worthwhile to review briefly some of the elements of interest associated with such systems. For one thing, they are much more ubiquitous in the real world. For another, they exhibit a much greater diversity of phases, and phase transitions often involve the rotational motions of molecules in interesting ways for which, of course, there is no counterpart in monatomic systems. As models of condensed matter, clusters are particularly convenient subjects for study. Solid van der Waals clusters of polyatomic molecules, for some reason, are able to adopt the structural organiza- tion of bulk crystal lattices spontaneously when they are one or two orders of magnitude smaller than van der Waals clusters of atoms that are able to grow as crystals. This means that molecular clusters can model bulk phases realistically when they are quite small, a fact compensating in some measure for the extra complexity of the potential functions required to represent the intermolecular interactions. That the results attain a certain degree of reality is confirmed by the close relationship they bear to results determined experimentally for clusters containing $10^{4} \mathrm{~mol}-$ ecules.

One of the compelling reasons for performing simulations on clusters rather than on bulk systems (where periodic boundary conditions are imposed) is that the phase transitions which various molecular clusters undergo spontaneously produce one or another of a variety of different structures. Such transformations occur freely in clusters but those involving a change of symmetry are severely inhibited when periodic boundary conditions are imposed. 


\section{Illustrative examples}

One of the most promising areas of application of MD simulations is the exploration of nucleation in phase changes in molecular clusters. Progress in this area, whether by experiment or by pure theory, has been frought with difficulty. Simulations, however, are beginning to reveal quite directly the development and growth of the critical nuclei that initiate the transitions. This is illustrated in Fig. 1 which displays three stages in a simulation by Chen [4] of the transformation of a cluster of 188 tert-butyl chloride molecules from an orientationally disordered tetragonal phase to an ordered monoclinic phase.

The MD simulations show in molecular detail how the transformation proceeds and yield important information about the kinetics of phase changes. They also illustrate how MD computations can supplement other techniques in crystallography. Before the simulation had been carried out, the neutron diffraction powder patterns for the colder phase had not been successfully indexed. The phase of cold tert-butyl chloride spontaneously generated in the MD simulation revealed the lattice parameters and crystal structure, and accounted quantitatively for the powder pattern [5].

Besides serving as model systems for condensed phases, clusters are of interest in the specific size effects they manifest on such properties as melting points and temperatures of other phase transitions. In the literature, this effect is almost universally rationalized in terms of the capillary theory $[6,7]$ which theory is also commonly incorporated into nucleation theory [8]. The idea in each case is that the free energy cost of generating a small particle of a lower-temperature phase in an undercooled higher-temperature phase is governed by the interfacial free energy of the boundary between the phases. Bulk-like interfacial free energies and enthalpies per volume are attributed to the phases. Results of the capillary theory are qualitatively reasonable but in very poor quantitative agreement with results of MD simulations [3,9]. Some of the reasons for the discrepancies are apparent in the following order-of-magnitude considerations.
Melting point depressions are inappreciable until particles fall below, say, $10^{4}$ to $10^{3}$ molecules, and critical nuclei in transformations are believed to contain only $10^{2}$ to $10^{1}$ molecules under common conditions. Furthermore, theoretical arguments $[10,11]$ suggest that liquid-vapor surface layers are several molecular layers thick and that transition layers between liquids and solids are even more diffuse, perhaps spanning 5 to 10 or more molecular layers. Now, implicit in the capillary model is a sharp, molecularly thin boundary between the phases. Quite apart from the expected (but theoretically uncertain) effect of surface curvature on interfacial free energy, there is a conflict between the basis of the capillary model and the small size of the very particles it has been introduced to treat. A little arithmetic makes the conflict evident. It is simple to show that a spherical particle of radius $R$ containing $N$ closely packed molecules of diameter $d$ has

$R / d \sim m^{-1}$

molecular layers from its center to its surface, and that the fraction $F$ of the particle's $N$ molecules that are in its surface, is

$F \sim 3 m(1-0.5 m)^{2}$

where

$m=(4 \pi / 3 N)^{1 / 3}$.

Results of applying Eqs. (1)-(3) are given in Table 1 .

Table 1 shows that the notion of small clusters being largely made up of interior molecules, with ample room inside their surface layers to harbor bulk-like cores surrounded by bulk-like solidliquid interfaces, is totally unrealistic if the esti-

Table 1

Fraction $F$ of molecules in an $N$-molecule cluster that are in the surface layer, and number of molecular layers $R / d$ between the center of a cluster and its surface

\begin{tabular}{lllll}
\hline$N$ & $10^{4}$ & $10^{3}$ & $10^{2}$ & $10^{1}$ \\
\hline$F$ & $\sim 0.21$ & $\sim 0.41$ & $\sim 0.71$ & $\sim 0.89$ \\
$R / d$ & $\sim 13$ & $\sim 6$ & $\sim 3$ & $\sim 1.5$ \\
\hline
\end{tabular}



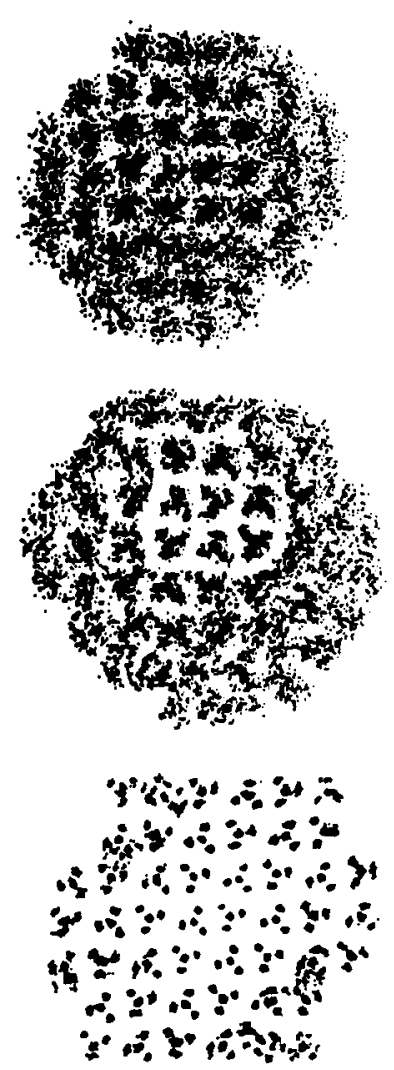

Fig. 1. Images of a crystalline cluster of 188 molecules of $\left(\mathrm{CH}_{3}\right)_{3} \mathrm{CCl}$ at various stages of cooling, looking down the molecular 3-fold $(\mathrm{C}-\mathrm{Cl})$ axis. Top, orientationally disordered tetragonal phase at $130 \mathrm{~K}$. Center, nucleus of monoclinic phase growing in the tetragonal phase at $80 \mathrm{~K}$. Right, ordered monoclinic phase at $50 \mathrm{~K}$ after transformation.

mates in Refs. [10] and [11] are correct. Be that as it may, the simulations imply that the diffuseness of the boundary layer between solid phases (see Fig. 1) may be appreciably lower than that expected for solid-liquid interfaces [10,11].

Molecular dynamics computations of clusters undergoing melting as they are heated confirm that the crispness of transition implied by the capillary model is not realized in simulations $[3,9]$. The melting, instead, is spread over a far wider temperature range than predicted by the capillary model, and the temperature at which the cluster is half-melted is not in close accord with that of the conventional theoretical treatment.
Table 1 also suggests a factor to keep in mind when analyzing nucleation rates derived from MD simulations of phase changes such as freezing when the nuclei of the new (colder) phase are wetted by the surrounding original phase. Such nuclei have always been seen to form in the interior of the cluster, and never on the surface. Inasmuch as the surface layer may constitute the major portion of the bulk of the cluster in a characteristic MD run, the active volume available for nucleation is much smaller than the total volume. This additional restriction limits further the validity of the capillary theory as a model for interpreting results.

Does the above account, then, imply that clusters are so atypical of condensed matter that they are useless as models of behavior in liquid and solid phases? The answer is that small molecular clusters emphasize just those aspects of behavior which are poorly accounted for by the standard capillary theory, yet they do possess the same phase structures and do undergo the same phase transitions that are found in large clusters and in bulk matter. Furthermore, in the process of nucleation, the critical nuclei are generally by their very nature too small to be well-described by capillary theory. Therefore, small molecular clusters do provide information relevant to the understanding of real systems, and simulations offer a particularly favorable opportunity for characterizing that behavior which has resisted treatment by alternative techniques.

\section{Acknowledgements}

This work was supported by a grant from the National Science Foundation. I thank J. Chen and $\mathrm{S}$. $\mathrm{Xu}$ for valuable discussions.

\section{References}

[1] See, for example, R.S. Berry, T.L. Beck, H.L. Davis and J. Jellinek, J. Adv. Chem. Phys. 70 (1988) 75.

[2] L.S. Bartell and S. Xu, J. Phys. Chem. 95 (1991) 8939.

[3] L.S. Bartell and J. Chen, J. Phys. Chem. 96 (1992) 8801.

[4] J. Chen and L.S. Bartell, J. Phys. Chem. 97 (1993) 10645. 
[5] E.J. Valente and B.M. Powell, B.M., unpublished research.

[6] J.J. Thomson, Applications of Chemical Dynamics, London, 1888.

[7] H. Reiss, P. Mirabel and R.L. Whetten, J. Phys. Chem. 92, (1988) 7241.
[8] D. Turnbull and J.C. Fisher, J. Chem. Phys. 17 (1949) 71; E.R. Buckle, Proc. Roy. Soc. London, A261 (1961) 189.

[9] S. Xu and L.S. Bartell, J. Phys. Chem. 97 (1993) 13544.

[10] X.C. Zeng and D.J. Stroud, J. Chem. Phys. 90 (1989) 5208.

[11] D. Oxtoby, Adv. Chem. Phys. 70 (1988) 263. 